\title{
NOTCH signalling in ovarian cancer angiogenesis
}

\author{
Jose Alejandro Perez-Fidalgo $^{1 \wedge}$, Belen Ortega ${ }^{1}$, Soraya Simon ${ }^{1}$, Eleftherios Pierre Samartzis ${ }^{2}$, \\ Stergios Boussios ${ }^{3,4,5}$
}

${ }^{1}$ Department of Medical Oncology, Hospital Clinico Universitario of Valencia, Biomedical Research Institute INCLIVA, CIBERONC, Valencia, Spain; ${ }^{2}$ Department of Gynecology, University Hospital Zurich, Zurich, Switzerland; ${ }^{3}$ King's College London, School of Medicine, Guy's Campus, London, UK; ${ }^{4}$ Medway NHS Foundation Trust, Gillingham, Kent, UK; ${ }^{5}$ AELIA Organization, 9th Km Thessaloniki-Thermi, Thessaloniki, Greece Contributions: (I) Conception and design: JA Perez-Fidalgo; (II) Administrative support: None; (III) Provision of study material or patients: None; (IV) Collection and assembly of data: None; (V) Data analysis and interpretation: None; (VI) Manuscript writing: All authors; (VII) Final approval of manuscript: All authors.

Correspondence to: Jose Alejandro Perez-Fidalgo. Department of Medical Oncology, Hospital Clinico Universitario of Valencia, Biomedical Research Institute INCLIVA, Valencia, Spain. Email: japfidalgo@msn.com.

\begin{abstract}
The Notch signalling pathway is involved in the new vessel formation process by regulating tip and stalk cells, which are key cells in the sprout formation. This process is essential in both normal ovary and cancer angiogenesis and is regulated by Notch-VEGF crosstalk. Furthermore, Notch has been linked in ovary with stem cell maintenance and epithelial mesenchymal transition processes. Dysregulation of the Notch pathway is frequent in ovarian cancer (OC) and it has been associated with impaired survival and advanced stages or lymph node involvement. Notch also plays a role in chemoresistance to platinum. In this context, this pathway has emerged as an attractive target for precision medicine in OC. Two main targets of this pathway concentrate the clinical development of compounds blocking Notch: gamma secretase and Delta-like ligand 4. Most of the clinical trials including OC patients have been developed in phase I or phase $\mathrm{Ib}$. Despite being in an early phase, both of these compounds, navicixizumab or demcizumab, two monoclonal antibodies targeting Dll4, showed promising efficacy data in platinum-resistant OC patients in recent studies. This review will focus on the mechanisms of the Notch pathway with special interest in angiogenesis regulation and the implication of Notch as a potential therapeutic target in OC.
\end{abstract}

Keywords: Ovarian cancer (OC); angiogenesis; Notch; Dll4; gamma-secretase

Submitted Jun 04, 2020. Accepted for publication Oct 28, 2020.

doi: $10.21037 /$ atm-20-4497

View this article at: http://dx.doi.org/10.21037/atm-20-4497

\section{Introduction}

Angiogenesis is a process by which new blood vessels will be created. The formation of new vessels is essential for tumour growth and proliferation. This process is mainly regulated by the vascular endothelial growth factor (VEGF) and its receptors (VEGFR 1-4). In ovarian cancer (OC), angiogenesis has become a crucial therapeutic target. Bevacizumab, an anti-VEGF-A, has shown activity from first line $(1,2)$ to platinum-resistant recurrence (3), while tirosinquinase inhibitors (TKI) with anti-VEGFR activity such as pazopanib or cediranib (4) have also shown activity in OC.

Notch signalling plays a crucial role in the regulation of the vascular homeostasis. By regulating the differentiation between different phenotypes of endothelial cells, Notch promotes the sprout formation, essential for the genesis of

$\wedge$ ORCID: 0000-0003-3568-4345 
new vessels.

Moreover, Notch signalling is also correlated with the acquisition and maintenance of cancer stem cell (CSC) properties in several tumours (5). The Notch pathway exerts oncogenic or tumour-suppressive effects depending on stage and subtype.

In OC Notch dysregulation is a common event. Notch 3 , one of the four Notch-receptors, has been reported to be overexpressed in $>20 \%$ of ovarian serous adenocarcinomas. It has been shown that deregulated expression of Notch 3 is correlated with tumour recurrence, resistance to chemotherapeutic agents, and an impaired outcome (6).

Multiple inhibitors and antagonistic monoclonal antibodies targeting Notch signalling components have been studied in preclinical and clinical trials (7). However, Notch-targeted agents have not yet been incorporated to the therapeutic armamentarium against OC.

In this review we will address the role of Notch signalling pathway in ovarian angiogenesis with particular focus on its therapeutic implications in the management of OC.

\section{An overview of the Notch pathway}

Notch is activated through cell-to-cell contact (paracrine communication) and serve as a communication mechanism with neighbouring cells. Notch pathway activation leads to cellular proliferation, differentiation, and/or apoptosis (8). As a consequence of this activation, the Notch pathway will control tissue homeostasis, playing an important role in angiogenesis regulation, and stem cell maintenance (9).

There are 4 Notch receptors (Notch 1-4) and 5 different ligands that have been identified in mammals (10). The ligands are the delta-like ligand family (Dll) 1,3 and 4 and the serrate like Jagged1 (JAG1) and Jagged2 (JAG2) ligands (11).

Receptors are transmembrane proteins with a Notch extracellular domain (NECD) that consist of multiple EGFR-like repeats, a transmembrane domain and a large Notch intracellular domain (NICD) containing a regulation of amino acid metabolisms that interacts with the transcription factor family C promoter (CBF-1)-Su-Lag1.

Dll4/JAG ligands regulate the proteolytic cleavage of Notch receptors by disintegrin and metalloproteinase domain-containing protein (ADAM) 10/17 and gamma secretase. These cleavage events release the NECD and NICD (12,13). The expression pattern of Dll4 and JAG modifies the strength of response of the Notch cascade (14).

NICD released by gamma-secretase action, is translocated to the nucleus, where it binds to the DNA bound CBF-1/ $\mathrm{Su}(\mathrm{H}) / \mathrm{Lag}-1$ protein complex (CSL complex) recruiting the MAML1-3 co-activators. As a result, the transcription of Notch target genes is initiated (9). These genes are transcription factors of Hes (Hairy/Enhancer of split) and Hey (Hes-related) families. These genes regulate the transcription of proteins involved in cell fate determination, being the downstream effectors of the Notch signalling, and they are involved in cancer progression, chemoresistance, self-renewal promotion, and tumour initiation in various cancers (15). Hes 1 locus is amplified in 2-25\% in OC, followed by Hey1, Notch2 and Notch3. See Figure 1.

The Notch signalling cascade response can be canonical or non-canonical. The canonical signalling cascade is the NICD/CSL-dependent transcription of Notch target genes (16), whereas the non-canonical cascade refers to the CSL-independent responses such as NICD-dependent inhibition of ATM or activation of RAC1 $(17,18)$.

The Notch pathway interacts with other signalling cascades, such as TGF-B, Wnt, EGFR and HER2 pathways, as well as the PI3K signalling cascade.

In adult tissue, Notch signalling maintains stem cells and controls tissue homeostasis. In fact, Notch signalling was first studied because of its implication in embriogenic development in mammals, where Notch has different implications in vascular and angiogenesis control such as proper cardiovascular development.

The Notch cascade is also related with cancer stemness being JAG1 the main ligand implicated. This process that regulates the ability of the cells to differentiate into different tumour cell types is crucial in cancer progression.

\section{The Notch pathway and angiogenesis in normal ovary}

The Notch pathway is related with the new vessel formation process through the differentiation of tip and stalk cells in the new sprout (19). Three types of cells are going to be involved in the formation of a new vessel: tip cells, stalk cells, and phalanx cells.

The tip cells navigate the environment via filopodia and response to vascular endothelial growth factor (VEGF). The stalk cells will form the vascular lumen, proliferate and undergo tubulogenesis. And finally phalanx cells, which are quiescent and support the sprout (20).

VEGF and Notch engage in a cross talk to balance tip and stalk cell formation. VEGF signalling through VEGF receptor 2 (VEGFR2) and neuropiline-1 (NRP1) (NRP1) 


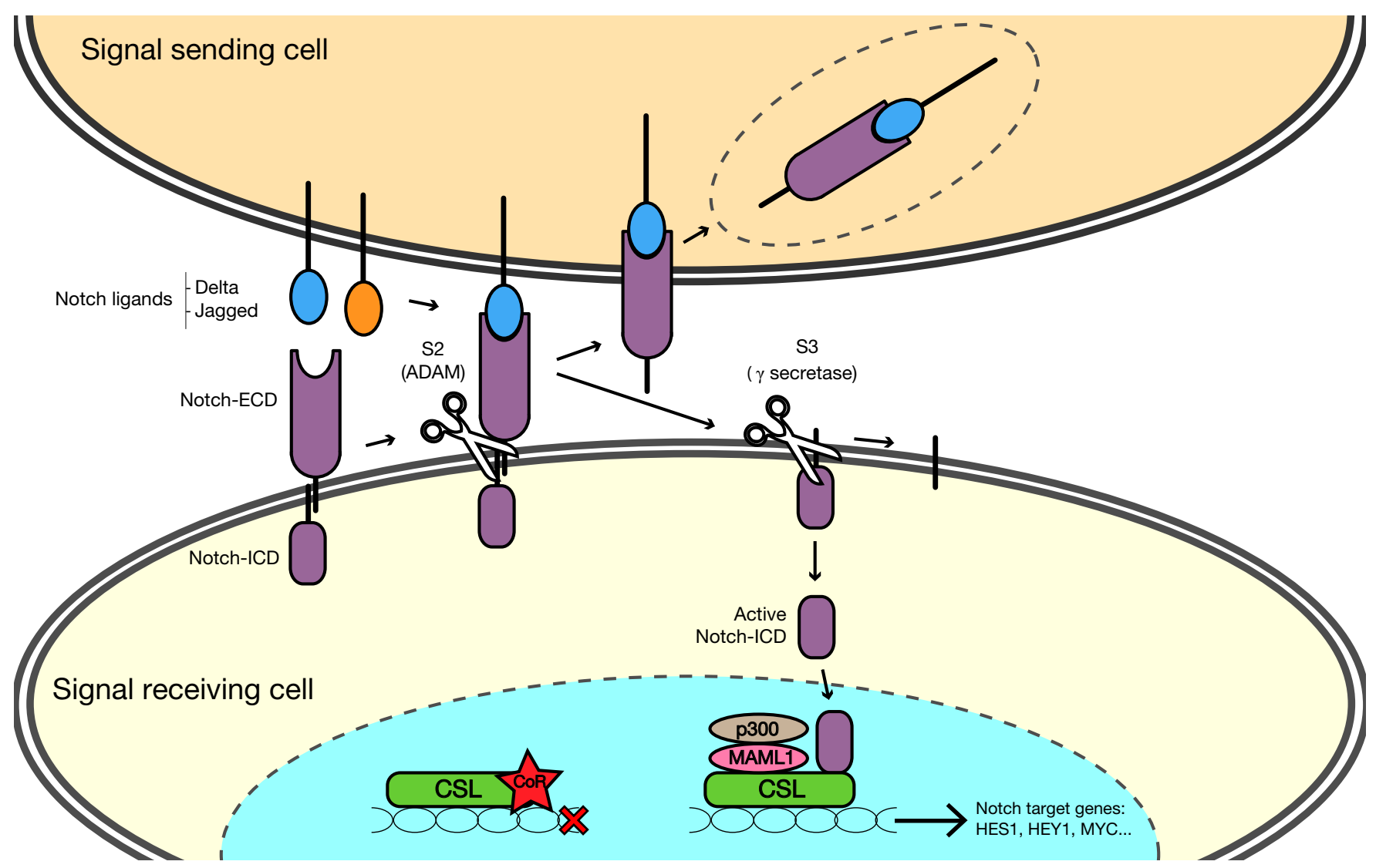

Figure 1 Notch signalling pathway. Interaction of the ligands Dll4 and Jagged with the Notch-ECD activates the secretases ADAM and gamma-secretase cleavage the two domains of Notch (ECD and ICD). Notch-ICD is released in the cytoplasm as an active form and translocates into the nucleus. In the nucleus Notch-ICD interacts with the CSL protein that in the absence of Notch-ID is inactivated by the a co-represor. Activated CSL forms a complex with proteins p300 and MAML1 that activates the transcription of Hes and Hey Notch related genes. ECD, extracellular domain. ICD, intracellular domain.

receptor on tip cells drives vascular sprouting and upregulates Dll4. Dll4 signalling through Notch receptors on endothelial stalk cells restricts angiogenic sprouting and proliferation through downregulation of VEGFR2 and NRP1 $(21,22)$.

Notch signalling upregulates JAG1 to antagonise the Dll4-dependet stalk phenotype leading to sprout formation and proliferation

In tumour cells JAG1 has been seen to be overexpressed being involved in tumour angiogenesis (23).

Dll4 is expressed in tumour blood vessels (tip cells) and stroma. When Dll4 activates Notch 1 receptors on the adjoining endothelial stalk cell, initiates a vascular VEGF negative feedback loop, which limitates sprouting by downregulation of VEGFR-2. In this context, Dll4 regulates VEGF-mediated vascular growth by preventing excessive branching that could potentially lead to vascular dysfunction (24).

In preclinical tumour models, inhibition of Dll4mediated Notch signalling in tumours results in hypersprouting of nonfunctional vasculature (23).

Epithelial ovarian cells express the JAG1, Dll1 and Dll4 ligands. Dll4 is expressed in the endothelium at sites of vascular development and angiogenesis (25). Fraser et al. confirmed in an in vivo experiment with primate ovaries that Dll4 blockade increases luteal angiogenesis and microvascular density (26). Other experiments in mice also showed that Notch signalling inhibited FGF-induced angiogenesis and blocked ovarian follicle development.

In summary the Notch pathway plays an essential role in vascular homeostasis and integrity in the normal ovary. 


\section{NOTCH and ovarian cancer}

The Notch pathway is frequently altered in microsatelliteinstable or POLE-mutant gastric and esophageal (79\%), colorectal $(70 \%)$ or endometrial cancers (64\%) (27). Moreover, Notch is also altered in leukemia, lymphoma, breast and lung cancers, among others (7). In OC, several studies have reported the critical roles of Notch components in cancer progression and metastasis.

The alteration of the Notch pathway genes has been reported in approximately $22 \%$ of all analysed ovarian tumours (15). A recent study found that JAG1 was overexpressed in OC cells, which stimulates the Notch pathway and promotes cell proliferation, migration, invasion, stemness, and chemoresistance (28).

VEGF, VEGFR1, Dll4, Notch1 or Notch3 in OC samples showed statistically significant higher expression than in normal ovary or than in benign ovarian tumour tissues. Dll4 expression was associated with higher expression of VEGFR1 and Notch1 with VEGFR2 and microvessel density suggesting the relevant role of Notch in ovarian angiogenesis (29).

In a recent analysis of several datasets including information from PRECOG, GENT, and CSIOVDB databases, the relevance of the Notch pathway in OC was assessed. The alteration of certain Notch-related genes has been associated with poorer prognostic factors and with an impaired survival in several studies (30). Notch-related genes were found to be differently expressed in OC and this overexpression was associated with poor overall survival (OS) and disease-free survival (DFS). High levels of Notch2, Notch3, and ADAM17 were associated with impaired OS and Notch2, Notch3, MAML1, and ADAM17 with poorer DFS.

Upregulation of Notch2, Notch3, DLL3, MAML1 and ADAM17 was associated with more advanced FIGO stages.

Notch3 expression has also been related with relapse in $\mathrm{OC}$ and was associated with impaired prognosis. Notch 3 expression by immunohistochemistry (IHC) was significantly higher in recurrent OC than in primary OC samples from the same patients. Moreover, high Notch3 nuclear protein or mRNA levels were related with impaired OS and progression-free survival (PFS) (31,32).

Ivan et al. (33), studied the DNA methylation patterns and miRNAs of the Notch family and its relationship with OS. The authors analysed the clinical data from gene expression and methylation data of more than 450 patients with advanced serous OC. Higher levels of CCND1, PPARG, and RUNX1 and lower levels of their respective
miRNAs were correlated with significantly poorer survival.

\section{NOTCH and ovarian cancer angiogenesis}

Angiogenesis is a key process in ovarian carcinogenesis. Wide clinical and preclinical evidence show that VEGFmediated angiogenesis is an important pathway in cancer and an anticancer target of outmost relevance. VEGF signalling activates several downstream effectors including Notch pathway in OC (34).

Experiments in mouse models (35) showed that Dll4 silence leads to a decrease in the angiogenesis and tumour growth that was synergistic with the addition of bevacizumab.

A Chinese study (29) in 28 samples from ovarian carcinomas showed that Dll4 was positively correlated with VEGFR1 expression, one of the transmembrane receptors of VEGF, whereas Notch1 was positively correlated with VEGFR2. In this study, VEGFR2 expression in ovarian tumours was associated with an impaired prognosis with more asicites and distant metastases.

In this context, the Notch pathway is not only related with an impaired survival in OC but it is also closely related with angiogenesis biomarkers in this tumour type.

\section{The synergistic effect of Notch targeting and anti-angiogenic treatment}

As previously mentioned, the Dll4-Notch pathway and VEGF regulate each other. D114 in endothelial cells is upregulated by VEGF (36). In fact, Dll4 expression in tumour vessels is rapidly decreased by blocking VEGF (37). It has also been seen that Notch signalling can alter expression of three VEGF receptors (38).

Hu et al. confirmed that blocking both Dll4 and VEGF with siRNA and bevacizumab respectively was more effective in inhibiting angiogenesis in preclinical models than either strategy. Moreover, exposure to anti-VEGF treatment leads tumours to underexpress Dll4 (39). In this context, a double blockade of VEGF and Dll4 could potentially be synergistic improving antitumour effect in OC.

\section{The Notch pathway implication in chemoresistance: the role of Notch3}

Although OC frequently responds to chemotherapy, most patients will develop a chemoresistant recurrence. In this 
context, approaches and specific biomarkers to resensitise patients to standard chemotherapies are a medical need (40).

Acquired resistance to antitumour therapies in response to repeated chemotherapy courses is a major challenge in the management of OC. However, the underlying mechanisms leading to chemoresistance in OC are poorly understood.

Notch3 pathway dysregulation is a frequent alteration in several tumours including OC (15).

Notch3 receptor has been identified as a potential oncogene and high levels of Notch3 have been found in approximately $20 \%$ of ovarian serous adenocarcinomas. Notch3 disregulation has been related with tumour recurrence, and drug resistance $(31,41)$.

Notch3 is overexpressed in aldehyde dehydrogenase isoform 1 (ALDH1+) ovarian cells (42) supporting a link between Notch3 and CSC. Studies by McAuliffe et al. (43) showed that Notch3 played a relevant role in CSC maintenance and resistance to platinum.

Kim et al. (42) observed that higher expression levels of ALDH1 and Notch related genes, and especially Notch3, were associated with CSCs and with chemoresistant ovarian SCs and paclitaxel-resistant SKpac OC cells. In addition, Notch3 expression correlated with poor prognosis factors, such as advanced stage, lymph node, and distant metastases.

Notch3 has also been related with epithelial mesenchymal transition (EMT). In a Canadian preclinical study, the OC cell line OVCA429 was transducted with an empty vector or a retroviral vector expressing Notch 3 intracellular domain or NICD3. Notch3 induced EMT in OVCA429 cells. Furthermore, OVCA429/NICD3 cell line showed higher resistance to carboplatin compared to the OVCA429 control cells (44).

Kang et al. studied the role of Notch3 specific inhibition in paclitaxel-resistant Skpac OC lines and parental SKOV3 cells. The study compared the effects on cell viability, migration, angiogenesis, cell cycle, and spheroid formation among others of either Notch3 siRNA or gamma secretase inhibitor (GSI). Notch3 silencing reversed paclitaxelresistance re-sensitising these cells to paclitaxel therapy. Similar effects were seen after exposure of cells to GSI. Notch knockdown also depleted OC stem cells with a spheroide formation reduction and downregulation of CSC markers (45).

A recent study by Ju-Yeong et al., identified that miRNA-136 directly targets Notch3. Mi-RNA-136 levels were significantly lower in ovarian serous carcinoma as compared with normal control tissues. The low expression of miR-136 was associated with poorer OS in OC patients.

The artificial miR-136 overexpression leads to a decrease in cell viability, proliferation, CSC spheroid formation, and angiogenesis. Moreover, miRNA-136 expression increased apoptosis in paclitaxel-resistant SKpac cells (46).

\section{The Notch pathway as therapeutic target: clinical development in ovarian cancer}

Notch targeting with small-molecule inhibitors or antibodies is currently under development in OC. Most important targets for Notch blockade are gamma-secretase and Dll4. However, other drugs such as the natural compounds xanthohumol (47) or withaferin A (48) were assessed in the preclinical setting showing inhibition of cell growth and cell cycle arrest in OC cell lines through downregulation of Notch1 and Notch3.

Inhibiting Notch is gaining interest and recent studies have developed this strategy in the clinic as single agent or in combination with chemotherapy. See Table 1. In the following chapters we will focus on those studies with clinical impact in OC.

\section{Targeting gamma-secretase}

Gamma-secretase induces a proteolytic release of the NICD receptor and also participates in a wide variety of cellular functions. Targeting this secretase will avoid the cleavage and release of NICD. Gamma-secretase inhibitors (GSI) affect both ligand and receptor interactions, decreasing tumour angiogenesis, due to the cumulative effect of inhibiting Notch signalling in the tumour as well as inhibiting both JAG1 and DLL4 on the vasculature. Several GSI compounds have been developed in recent years.

A preclinical study by Chen $e t a l$. analysed the effects of MK-0752 alone or in combination with cisplatin in OC. MK-0752 alone induced cell growth inhibition, cell cycle arrest and apoptosis through downregulation of Notch 1 and its downstream effectors including Hes1, XIAP, c-Myc, and MDM2. Combination of sequential cisplatin followed by MK-0752 significantly increased apoptosis and inhibited the subcutaneous xenograft growth of OC in nude mice (49).

Bocchicchio et al. studied the effect of DAPT (a gamma secretase enzyme complex inhibitor) compared to the inhibition of B-catenin and their combination. This study showed that both pathways cooperated ensuring proliferation and migration of cells. Moreover, an upregulation of the Notch pathway in the absence of Wnt 
Table 1 Clinical studies of compounds targeting Notch pathway in ovarian cancer

\begin{tabular}{|c|c|c|c|c|c|c|}
\hline Compound & Target & Type of drug & Phase & $\mathrm{N}$ (all pts) & $\begin{array}{l}\text { Ovarian } \\
\text { cancer pts }\end{array}$ & Comments \\
\hline MK-0752 & GSI & Inhibitor & I & 103 & 3 & Recommended doses 1,800 to 4,200 mg weekly \\
\hline LY900009 & GSI & Inhibitor & I & 35 & 11 & Recommended dose $30 \mathrm{mg} 3$ times a week \\
\hline $\begin{array}{l}\text { Crenigascestat } \\
\text { (LY3039478) }\end{array}$ & GSI & Inhibitor & I & 110 & 10 & $\begin{array}{l}\text { Well tolerated at doses with target engagement. } \\
\text { Signs of activity }\end{array}$ \\
\hline \multirow{2}{*}{ RO4929097 } & \multirow{2}{*}{ GSI } & \multirow{2}{*}{ Inhibitor } & Ib (+ temsi) & 17 & 1 & $\begin{array}{l}\text { RP2D for RO } 20 \mathrm{mg}+\text { temsi } 37.5 \mathrm{mg} \text {. } \\
\text { Combination tolerable }\end{array}$ \\
\hline & & & II & 45 & $45 \mathrm{PROC}$ & Insufficient activity as single agent \\
\hline \multirow{2}{*}{$\begin{array}{l}\text { Navicixizumab } \\
\text { (OMP-305B83) }\end{array}$} & \multirow{2}{*}{$\begin{array}{l}\text { Anti DII4 } \\
\text { and VEGF }\end{array}$} & \multirow{2}{*}{$\begin{array}{l}\text { Dual Monoclonal } \\
\text { antibody }\end{array}$} & & 66 & 12 & $25 \%$ responses in ovarian cancer \\
\hline & & & lb (+ wpacl) & 18 & $18 \mathrm{PROC}$ & 43\% PR. 77\% CBR. Median PFS 7.3 months \\
\hline \multirow{2}{*}{$\begin{array}{l}\text { Demcizumab } \\
\text { (OMP-21M18) }\end{array}$} & \multirow[t]{2}{*}{ Anti DII4 } & \multirow{2}{*}{$\begin{array}{l}\text { Monoclonal } \\
\text { antibody }\end{array}$} & I & 55 & $1 \mathrm{OGCT}$ & Demcizumab well tolerated. Signs of activity \\
\hline & & & $\mathrm{lb}(+$ wpacl) & 19 & 19 PROC & $21 \%$ ORR. $43 \%$ CBR \\
\hline
\end{tabular}

pts, patients; GSI, gamma secretase inhibitor; DII4, delta-like ligand 4; RP2D, recommended phase 2 dose; gemc, gemcitabine; temsi, temsirolimus; wpacl, weekly paclitaxel; PROC, platinum-resistant ovarian cancer; OGCT, ovarian granulosa cell tumor; PR, parcial response; ORR, overall response rate; CBR, clinical benefit rate.

signalling suggested a crosstalk between the WNT pathway and Notch (50).

In the clinical setting different GSI have been tested in early phase trials. MK-0752 was explored a phase I trial in adult patients with advanced solid tumours. The study included 103 patients of whom 3 were OC patients. Significant inhibition of Notch signalling was observed with the 1800 to $4200 \mathrm{mg}$ weekly dose levels, confirming target engagement at those doses. One patient achieved a response and 10 a stable disease (51).

LY900009 also known as crenigacestat, is an oral GSI that has been assessed in a first-in-human phase I trial. A first part of dose escalation recruited patients in cohorts of 3 patients with advanced solid tumour and a second part for dose confirmation enrolled only OC patients.

Thirty-five patients were included. Most common adverse events (AE) were diarrhea (46\%), vomiting (34\%), anorexia (31\%), and nausea (31\%). In terms of efficacy, 5 patients had stable disease (SD) as best response.

Two patients $(5.7 \%)$ with leiomyosarcoma and OC received 4 courses of LY900009. The recommended MTD schedule was $30 \mathrm{mg}$ twice weekly.

Massard et al. studied the safety, pharmacokinetic profile, pharmacodynamic effects, and antitumour activity of crenigacestat, in patients with advanced or metastatic cancer in a phase I study. Most common adverse events were gastrointestinal. The study included 110 patients, and 10 patients with OC. This potent inhibitor of Notch signalling was well tolerated and demonstrated clinical activity in heavily pretreated patients (52).

Richter et al., conducted a phase I trial of an oral GSI, RO4929097 in combination with gemcitabine. Patients with advanced solid tumours were included. This study showed promising activity and identified a potential predictive biomarker, as lower levels of Notch protein expression were related with an increased benefit $(53,54)$.

In a phase Ib trial, RO429097 was assessed in combination with temsirolimus in refractory advanced solid tumours. The combination was tolerable with two DLTs; grade 3 rash and grade 3 mucositis in the same patient. The recommended phase 2 dose (RP2D) was established in $20 \mathrm{mg}$ for RO4929097 + $37.5 \mathrm{mg}$ for temsirolimus (55). 
RO4929097 has been further developed in a phase II trial in OC patients. 45 women with platinum-resistant OC and treated with a maximum of 2 previous regimens were enrolled. Patients received oral RO4929097 at $20 \mathrm{mg}$ 3 days on 4 days off per week in a 21 -day cycle. The primary endpoint was PFS rate at the end of 4 cycles. No objective responses were observed and 15 patients (33\%) had SD. The median PFS was 1.3 months (1.2-2.5 months). Treatment was well tolerated. Approximately $10 \%$ of patients discontinued treatment due to $\mathrm{AE}$. In high grade serous OC, the level of NICD protein by IHC was identified as a potential biomarker for longer PFS. NICD high levels showed a trend towards longer PFS than lower levels (3.3 vs. 1.3 months, $\mathrm{P}=0.09$ ). The drug showed insufficient activity as single agent to warrant further development in platinum-resistant OC (56).

Toxicity is a major concern in GSI treatment. A common AE is gastrointestinal toxicity and diarrhea, which is secondary to goblet cell metaplasia of small intestine attributed to the dual inhibition of Notch1 and Notch3 (57).

\section{Targeting Dll4}

Compared to GSI, Dll4 targeting leads to a massive but non productive angiogenesis instead of a reduced angiogenesis (37).

Kuhnert et al. (58), published the results of a preclinical study with REGN421 (enoticumab), an intravenous human IgG1 monoclonal antibody that binds Dll4, resulting in the inhibition of Notch pathway in OC models.

Enocitumab was administered to human tumour xenografts in immunodeficient mice engineered to express human Dll4. Exposure to enocitumab led to tumour growth inhibition and formation of nonfunctional tumour blood vessels. The antitumour effects were significantly enhanced when inhibition of VEGF with aflibercept was used simultaneously with Dll4 blockade.

Another study assessed the combination of double inhibition Dll4 and VEGF. An American group specifically assessed the role of the combination of Dll4 inhibition with the murine antibody enocitumab and the human antibody enocitumab with aflibercept or chemotherapy in orthotopic mouse models of OC. Double target of VEGF and D114 with enocitumab showed superior antitumour effects than either monotherapy. This study confirmed the therapeutic potential of the double blockade of VEGF and D114 (59).

Enocitumab was developed in the clinical setting in a phase I first-in-human trial. Enocitumab was administered with dose escalation from 0.25 to 4 $\mathrm{mg} / \mathrm{kg}$ triweekly (Q3W) and 0.75 to $3 \mathrm{mg} / \mathrm{kg}$ every 2 weeks $(\mathrm{Q} 2 \mathrm{~W})$. Of 53 patients included, 31 were treated Q3W and 22 Q2W. An MTD was not reached. Two DLTs occurred: grade 3 nausea and grade 3 abdominal pain. Antitumour activity included two partial responses (one in $\mathrm{OC}$ ) and 16 patients with $\mathrm{SD}$, of whom 3 lasted more than 6 months. Enoticumab was tolerated with a RP2D of $4 \mathrm{mg} / \mathrm{kg}$ Q3W and $3 \mathrm{mg} / \mathrm{kg}$ Q2w based on pharmacokinetics and clinical activity (60).

OMP-305B83 also knows as navicixizumab, is a bispecific anti-Dll4 and anti-VEGF dual antibody. Jimeno et al., (61) assessed at the first-in-human phase 1a study, the role of navicixizumab in previously treated solid tumours. Study objectives were the determination of MTD, safety, and efficacy, among others. Sixty-six patients were treated once every 3 weeks in 8 dose-escalation cohorts and an expansion cohort. The most commonly enrolled tumour type was OC (12) followed by colorectal (11), breast, pancreatic and endometrial cancers (4 each). MTD was not reached (only 1 DLT occurred). Most frequent treatment-related AE were hypertension $(57.6 \%)$, headache $(28.8 \%)$ and fatigue (25.8\%). Pulmonary hypertension was mostly asymptomatic at doses $\leq 5 \mathrm{mg} / \mathrm{kg}$ but was more severe at higher dosis with one patient with grade 3 . Regarding efficacy, 4 patients (3 OC, 1 uterine carcinosarcoma) had a partial response (PR) and 17 patients had SD. Of note, 19 patients had a reduction in the size of their target lesions including 4 responses of 12 patients with OC (25\%). In this context OMP-305B83was tolerable and showed promising activity mainly in OC.

Navicixizumab had been further developed in combination with weekly paclitaxel in OC patients by Fu et al. Preliminary data of a phase Ib trial assessed this combination and were presented in ESMO 2018 (62). Eighteen patients with platinum-resistant OC treated with $>2$ prior therapies and/or bevacizumab were included. Paclitaxel was given on days 1,8 , and 15 and navicixizumab on days 1 and 15 of every 28-day cycle. The study had a dose escalation design testing OMP-305B83 doses of 3 or 4 $\mathrm{mg} / \mathrm{kg}$ followed by an expansion cohort.

The efficacy data of navicixizumab shown in heavily pretreated platinum resistant OC patients was encouraging and led the FDA on October 2019 to grant a fast track designation to navicixizumab for the treatment of patients with high-grade ovarian, primary peritoneal or fallopian tube cancer who have received $\geq 3$ prior therapies and/or 
prior bevacizumab.

In SGO 2020 the interim efficacy analysis of the Fu $\mathrm{S}$. phase Ib study of navicixizumab in combination with paclitaxel confirmed important activity. At that time 44 patients had been included. The overall response rate (ORR) of this combination per RECIST 1.1 was $43 \%(n=19)$ including 1 complete and 18 PR. Fifteen (34\%) patients had $\mathrm{SD}$ as best response with a clinical benefit rate (CBR) of $77 \%$. The median time to progression was 7.3 months and the median duration of response was 5.7 months. The safety profile appeared to be manageable with hypertension being the most common $\mathrm{AE}$.

Demcizumab (OMP-21M18) is an IG2 humanized monoclonal antibody that targets Dll4 leading to a potent inhibition of the Notch pathway.

A phase I trial evaluated the safety, pharmacokineics, and pharmacodynamics of demcizumab in patients with advanced malignancies. Patients were treated with 5 different dose and schedules. Fifty-five patients were treated but no more than one DLT was seen at any dose level. The MTD was not reached for either schedule. Most frequent AEs were hypertension $(47 \%)$, fatigue $(31 \%)$, anaemia $(22 \%)$ and headache $(20 \%)$. Five patients at different dose levels developed different grades of heart failure and other five were hospitalised due to bleeding episodes. Sixteen of 25 evaluable patients (64\%) had stabilization of disease or response (63).

A recent phase Ib trial, the SIERRA trial, assessed the MTD or the maximum administered dose and safety of the combination of demcizumab and paclitaxel in platinumresistant OC patients. Patients included should have received no more than 4 prior chemotherapy regimens. Following a $3+3$ design, demcizumab was administered at different dose levels on days 1 and 15 in combination with weekly paclitaxel every 28 days. The doublet was administered for a maximum of 3 cycles continuing paclitaxel monotherapy until unacceptable toxicity or disease progression. The study included 19 patients. No DLTs were observed, and thus the MTD was not reached. The side effects with a frequency over $50 \%$ were diarrhea, fatigue, peripheral edema, and nausea. Pulmonary hypertension grade 2 in two patients and grade 1 in another patient was also observed. The ORR was $21 \%$ (95\% CI: 6-45\%) and the CBR was $43 \%$ (95\% CI: $20-66 \%$ ). In conclusion, the combination of demcizumab and paclitaxel showed an acceptable toxic profile and activity in a heavily pretreated platinum-resistant cohort (64).

\section{Conclusions}

The Notch pathway plays a crucial role in OC and has an impact on prognosis. Notch and VEGF are essential in OC angiogenesis and Notch has also been related with chemoresistance. Thus, Notch targeting, and mainly dual targeting of Notch and VEGF, is a promising strategy in OC. The combination of Notch inhibition with chemotherapy or antiangiogenics showed interesting activity in early phase clinical studies. Navicixizumab, a dual anti-Dll4 and anti-VEGF in combination with weekly paclitaxel showed a response rate of $43 \%$ in heavily pretreated platinum-resistant OC patients. This combination has received the first FDA fast track designation for a Notch inhibitor in OC. Although toxicity is manageable, gastrointestinal AEs and hypertension could be important. There is a need of further clinical trials in order to confirm the early signs of activity and to identify a potential biomarker of activity for GSI or Dll4 blockade.

\section{Acknowledgments}

Funding: None.

\section{Footnote}

Provenance and Peer Review: This article was commissioned by the editorial office, Annals of Translational Medicine for the series "Ovarian Cancer: State of the Art and Perspectives of Clinical Research". The article has undergone external peer review.

Conflicts of Interest: All authors have completed the ICMJE uniform disclosure form (available at http://dx.doi. org/10.21037/atm-20-4497). The series "Ovarian Cancer: State of the Art and Perspectives of Clinical Research" was commissioned by the editorial office without any funding or sponsorship. SB served as the unpaid Guest Editor of the series and serves as an unpaid editorial board member of Annals of Translational Medicine from Nov 2019 to Oct 2021. Dr. JAPF reports grants from Mutua Madrileña, grants from Sociedad Española de Oncologia Medica (Spanish Society of Medical Oncology), during the conduct of the study; personal fees from AstraZeneca, personal fees from GSK - Tesaro, personal fees from Clovis, personal fees from 
Roche, personal fees from amgem, personal fees from Pfizer, personal fees from Pharmamar, outside the submitted work; in addition, Dr. JAPF has a patent Predictive signature for neoadjuvant chemotherapy in breast cancer pending. The authors have no other conflicts of interest to declare.

Ethical Statement: The authors are accountable for all aspects of the work ensuring that questions related to the accuracy or integrity of any part of the work are appropriately investigated and resolved.

Open Access Statement: This is an Open Access article distributed in accordance with the Creative Commons Attribution-NonCommercial-NoDerivs 4.0 International License (CC BY-NC-ND 4.0), which permits the noncommercial replication and distribution of the article with the strict proviso that no changes or edits are made and the original work is properly cited (including links to both the formal publication through the relevant DOI and the license). See: https://creativecommons.org/licenses/by-nc-nd/4.0/.

\section{References}

1. Tewari KS, Burger RA, Enserro D, et al. Final overall survival of a randomized trial of bevacizumab for primary treatment of ovarian cancer. J Clin Oncol 2019;37:2317-28.

2. Perren TJ, Swart AM, Pfisterer J, et al. A phase 3 trial of bevacizumab in ovarian cancer. $\mathrm{N}$ Engl J Med 2011;365:2484-96.

3. Pujade-Lauraine E, Hilpert F, Weber B, et al. Bevacizumab combined with chemotherapy for platinum-resistant recurrent ovarian cancer: The AURELIA open-label randomized phase III trial. J Clin Oncol 2014; 32:1302-8.

4. Ledermann JA, Embleton AC, Raja F, et al. Cediranib in patients with relapsed platinum-sensitive ovarian cancer (ICON6): A randomised, double-blind, placebo-controlled phase 3 trial. Lancet 2016; 387:1066-74.

5. Ranganathan P, Weaver KL, Capobianco AJ. Notch signalling in solid tumours: A little bit of everything but not all the time. Nat Rev Cancer 2011;11:338-51.

6. Ceccarelli S, Megiorni F, Bellavia D, et al. Notch3 targeting: A novel Weapon Against Ovarian cancer stem cells. Stem Cell Int 2019:6264931.

7. Katoh M, Katoh M. Precision medicine for human cancers with Notch signaling dysregulation. Int J Mol Med 2020;45:279-97.

8. Perdigoto CN, Bardin AJ. Sending the right signal: Notch and stem cells. Biochim Biophys Acta 2013;1830:2307-22.
9. Groeneweg JW, Foster R, Growdon WB, et al. Notch signaling in serous ovarian cancer. J Ovarian Res 2014;7:95-100.

10. Bray SJ. Notch signalling: A simple pathway becomes complex. Nat Rev Mol Cell Biol 2006;7:678-89.

11. Rose SL. Notch signaling pathway in ovarian cancer. Int J Gynecol Cancer 2009;19:564-6.

12. Lambrecht BN, Vanderkerken M, Hammad H. The emerging role of ADAM metalloproteinases in immunity. Nat Rev Immunol 2018;18:745-58.

13. Yang G, Zhou R, Zhou Q, et al. Structural basis of Notch recognition by human $\gamma$-secretase. Nature 2019;565:192-7.

14. Sjöqvist M, Andersson ER. Do as I say, Not(ch) as I do: Lateral control of cell fate. Dev Biol 2019;447:58-70.

15. Cancer Genome Atlas Research Network. Integrated genomic analyses of ovarian carcinoma. Nature 2011;474:609-15.

16. Kopan R, Ilagan MXG. The Canonical Notch Signaling Pathway: Unfolding the Activation Mechanism. Cell 2009;137:216-33.

17. Nandagopal N, Santat LA, LeBon L, et al. Dynamic Ligand Discrimination in the Notch Signaling Pathway. Cell 2018;172:869-80.e19.

18. Bray SJ, Gomez-Lamarca M. Notch after cleavage. Curr Opin Cell Biol 2018;51:103-109.

19. Xie Q, Cheng Z, Chen X, et al. The role of Notch signalling in ovarian angiogenesis. J Ovarian Res 2017;10:13.

20. Norton KA, Popel AS. Effects of endothelial cell proliferation and migration rates in a computational model of sprouting angiogenesis. Sci Rep 2016;6:36992.

21. Potente M, Gerhardt H, Carmeliet P. Basic and therapeutic aspects of angiogenesis. Cell 2011;146:873-87.

22. Goel HL, Mercurio AM. VEGF targets the tumour cell. Nat Rev Cancer 2013;13:871-82.

23. Dufraine J, Funahashi Y, Kitajewski J. Notch signaling regulates tumor angiogenesis by diverse mechanisms. Oncogene 2008;27:5132-7.

24. Hellström M, Phng LK, Hofmann JJ, et al. Dll4 signalling through Notch1 regulates formation of tip cells during angiogenesis. Nature 2007; 445:776-80.

25. Choi HJ, Armaiz Pena GN, Pradeep S, et al. Anti-vascular therapies in ovarian cancer: moving beyond anti-VEGF approaches. Cancer Metastasis Rev 2015;34:19-40.

26. Fraser HM, Hastings JM, Allan D, et al. Inhibition of delta-like ligand 4 induces luteal hypervascularization followed by functional and structural luteolysis in the primate ovary. Endocrinology 2012;153:1972-83. 
27. Sanchez-Vega F, Mina M, Armenia J, et al. Oncogenic Signaling Pathways in The Cancer Genome Atlas. Cell 2018;173:321-37.e10.

28. Liu Z, Zhu Y, Li F, Xie Y. GATA1-regulated JAG1 promotes ovarian cancer progression by activating Notch signal pathway. Protoplasma 2020;257:901-10.

29. Wang H, Huang X, Zhang J, et al. The expression of VEGF and Dll4/Notch pathway molecules in ovarian cancer. Clin Chim Acta 2014;436:243-8.

30. Jia D, Underwood J, Xu Q, et al. Notch2/notch3/dll3/ maml1/adam17 signaling network is associated with ovarian cancer. Oncol Lett 2019;17:4914-20.

31. Park JT, Chen X, Tropè CG, et al. Notch3 overexpression is related to the recurrence of ovarian cancer and confers resistance to carboplatin. Am J Pathol 2010;177:1087-94.

32. Rahman MT, Nakayama K, Rahman M, et al. Notch3 overexpression as potential therapeutic target in advanced stage chemoresistant ovarian cancer. Am J Clin Pathol 2012;138:535-44.

33. Ivan C, Hu W, Bottsford-Miller J, et al. Epigenetic analysis of the Notch superfamily in high-grade serous ovarian cancer. Gynecol Oncol 2013;128:506-11.

34. Liu Z, Fan F, Wang A, et al. Dll4-Notch signaling in regulation of tumor angiogenesis. J Cancer Res Clin Oncol 2014;140:525-36.

35. Uyttendaele H, Ho J, Rossant J, Kitajewski J. Vascular patterning defects associated with expression of activated Notch4 in embryonic endothelium. PNAS 2001;98:5643-48.

36. Patel NS, Li JL, Generali D, et al. Up-regulation of deltalike 4 ligand in human tumor vasculature and the role of basal expression in endothelial cell function. Cancer Res 2005;65:8690-97.

37. Noguera-Troise I, Daly C, Papadopoulos NJ, et al. Blockade of Dll4 inhibits tumour growth by promoting non-productive angiogenesis. Nature 2006;444:1032-7.

38. Suchting S, Freitas C, Toro R del, et al. The Notch ligand Delta-like 4 (D114) negatively regulates endothelial tip cell formation and vessel branching. Proc Natl Acad Sci U S A 2007;104:3225-30.

39. $\mathrm{Hu} \mathrm{W}, \mathrm{Lu} \mathrm{C}, \mathrm{Han} \mathrm{HD}$, et al. Biological roles of the delta family notch ligand D114 in tumor and endothelial cells in ovarian cancer. Cancer Res 2011;71:6030-9.

40. Christie EL, Bowtell DDL. Acquired chemotherapy resistance in ovarian cancer. Ann Oncol 2017;28:viii13viii15.

41. Park JT, Li M, Nakayama K, et al. Notch3 gene amplification in ovarian cancer. Cancer Res
2006;66:6312-8.

42. Kim MJ, Kim AR, Jeong JY, et al. Correlation of ALDH1 and Notch3 expression: Clinical implication in ovarian carcinomas. J Cancer 2017;8:3331-42.

43. McAuliffe SM, Morgan SL, Wyant GA, et al. Targeting Notch, a key pathway for ovarian cancer stem cells, sensitizes tumors to platinum therapy. PNAS 2012;109:E2939-48.

44. Gupta N, Xu Z, El-Sehemy A, et al. Notch3 induces epithelial-mesenchymal transition and attenuates carboplatin-induced apoptosis in ovarian cancer cells. Gynecol Oncol 2013;130:200-6.

45. Kang H, Jeong JY, Song JY, et al. Notch3-specific inhibition using siRNA knockdown or GSI sensitizes paclitaxel-resistant ovarian cancer cells. Mol Carcinog 2016;55:1196-209.

46. Jeong JY, Kang H, Kim TH, et al. MicroRNA-136 inhibits cancer stem cell activity and enhances the anti-tumor effect of paclitaxel against chemoresistant ovarian cancer cells by targeting Notch3. Cancer Lett 2017;386:168-78.

47. Drenzek JG, Seiler NL, Jaskula-Sztul R, et al.

Xanthohumol decreases Notch1 expression and cell growth by cell cycle arrest and induction of apoptosis in epithelial ovarian cancer cell lines. Gynecol Oncol 2011;122:396-401.

48. Zhang X, Samadi AK, Roby KF, et al. Inhibition of cell growth and induction of apoptosis in ovarian carcinoma cell lines $\mathrm{CaOV} 3$ and SKOV3 by natural withanolide Withaferin A. Gynecol Oncol 2012;124:606-12.

49. Chen X, Gong L, Ou R, Zheng Z, Chen J, Xie F, et al. Sequential combination therapy of ovarian cancer with cisplatin and $\gamma$-secretase inhibitor MK-0752. Gynecol Oncol 2016;140:537-44.

50. Bocchicchio S, Tesone M, Irusta G. Convergence of Wnt and Notch signaling controls ovarian cancer cell survival. J Cell Physiol 2019;234:22130-43.

51. Krop I, Demuth T, Guthrie T, et al. Phase I pharmacologic and pharmacodynamic study of the gamma secretase (Notch) inhibitor MK-0752 in adult patients with advanced solid tumors. J Clin Oncol 2012;30:2307-13.

52. Massard C, Azaro A, Soria JC, et al. First-in-human study of LY3039478, an oral Notch signaling inhibitor in advanced or metastatic cancer. Ann Oncol 2018;29:1911-7.

53. Richter S, Bedard PL, Chen EX, et al. A phase i study of the oral gamma secretase inhibitor R04929097 in combination with gemcitabine in patients with advanced solid tumors (PHL-078/CTEP 8575). Invest New Drugs 2014;32:243-9.

54. van Es JH, Van Gijn ME, Riccio O, et al. Notch/ $\gamma$-secretase 
inhibition turns proliferative cells in intestinal crypts and adenomas into goblet cells. Nature 2005;435:959-63.

55. Diaz-Padilla I, Hirte H, Oza AM, et al. A phase Ib combination study of RO4929097, a gamma-secretase inhibitor, and temsirolimus in patients with advanced solid tumors. Invest New Drugs 2013;31:1182-91.

56. Diaz-Padilla I, Wilson MK, Clarke BA, et al. A phase II study of single-agent RO4929097, a gamma-secretase inhibitor of Notch signaling, in patients with recurrent platinum-resistant epithelial ovarian cancer: A study of the Princess Margaret, Chicago and California phase II consortia. Gynecol Oncol 2015;137:216-22.

57. Espinoza I, Miele L. Notch inhibitors for cancer treatment. Pharmacol Ther 2013;139:95-110.

58. Kuhnert F, Chen G, Coetzee S, et al. Dll4 Blockade in Stromal Cells Mediates Antitumor Effects in Preclinical Models of Ovarian Cancer. Cancer Res 2015;75:4086-96.

59. Huang J, Hu W, Hu L, et al. Dll4 Inhibition plus Aflibercept markedly reduces ovarian tumor growth. Mol Cancer Ther 2016;15:1344-52.

60. Chiorean EG, LoRusso P, Strother RM, et al. A phase I first-in-human study of enoticumab (REGN421), a fully

Cite this article as: Perez-Fidalgo JA, Ortega B, Simon S, Samartzis EP, Boussios S. NOTCH signalling in ovarian cancer angiogenesis. Ann Transl Med 2020;8(24):1705. doi: 10.21037/atm$20-4497$ human delta-like ligand 4 (D114) monoclonal antibody in patients with advanced solid tumors. Clin Cancer Res 2015;21:2695-703.

61. Jimeno A, Moore KN, Gordon M, et al. A first-inhuman phase 1a study of the bispecific anti-DLL4/antiVEGF antibody navicixizumab (OMP-305B83) in patients with previously treated solid tumors. Invest New Drugs 2019;37:461-72.

62. Fu S, Corr B, Hamilton E, et al. A phase Ib study of navicixizumab \& weekly paclitaxel in heavily pre-treated platinum resistant ovarian, primary peritoneal or fallopian tube cancer. Ann Oncol 2018;29:viii332-viii358.

63. Smith DC, Eisenberg PD, Manikhas G, et al. A phase I dose escalation and expansion study of the anticancer stem cell agent demcizumab (Anti-DLL4) in patients with previously treated solid tumors. Clin Cancer Res 2014;20:6295-303.

64. Coleman RL, Handley KF, Burger R, et al. Demcizumab combined with paclitaxel for platinum-resistant ovarian, primary peritoneal, and fallopian tube cancer: The SIERRA open-label phase Ib trial. Gynecol Oncol 2020;157:386-91. 